\title{
Intracerebral hemorrhage in a young man
}

\author{
Shelagh B. Coutts MD, Uta Matysiak-Scholze PhD, Jürgen Kohlhase MD, A. Micheil Innes MD
}
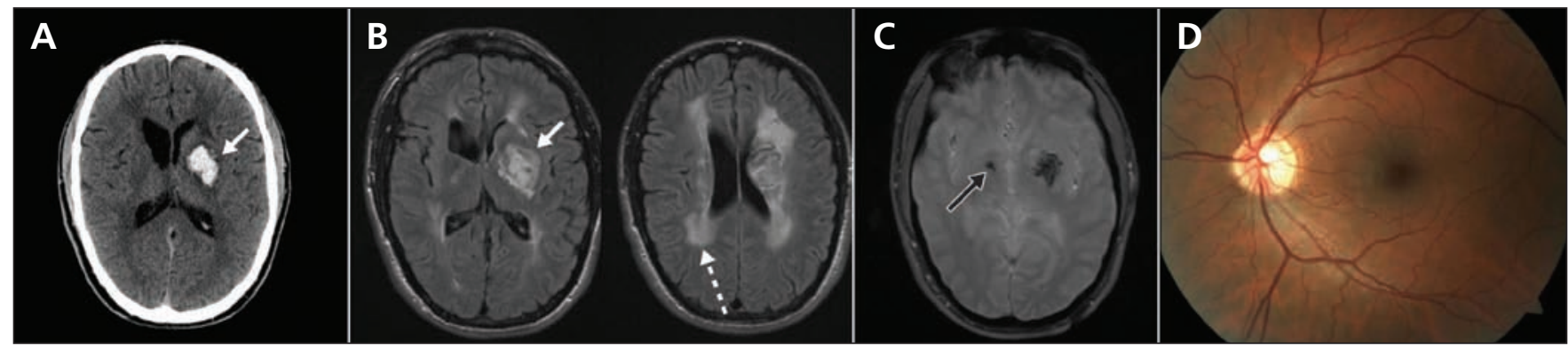

Figure 1: (A) Computed tomographic scan and (B) magnetic resonance images (with fluid attenuated inversion recovery pulse sequences) of the brain of a 27-year-old man showing representative slices of the hemorrhage in the left basal ganglia (solid white arrows). The magnetic resonance images show diffuse white matter hyperintensity within the deep white matter of both cerebral hemispheres (dashed arrow). (C) Gradient echo image showing dark areas, which represent the acute deep hemorrhage and the presence of previous hemorrhages that had been clinically asymptomatic (black arrow). (D) Retinal photograph of the left eye showing tortuous retinal vessels.

$\mathrm{W}$ hile driving to work, a 27-year-old man had a motor vehicle collision; when the police attended the scene they found him to be drowsy and immediately called an ambulance. The man, who was pre viously healthy, presented with slurred speech and rightsided weakness. Earlier that day, he had complained to his mother that he wasn't feeling well, and some mild heaviness and numbness had developed on his right side.

The patient denied any injury to the head from the collision. His vital signs were within normal limits, with a blood pressure of $115 / 59 \mathrm{~mm} \mathrm{Hg}$. The patient was drowsy. There was evidence of moderate right-sided hemiparesis, mild reduction in sensation to pinprick on the right, mild receptive and expressive dysphasia, and severe dysarthria.

The patient had no significant medical history, and he was taking no medications. He smoked cigarettes and occasionally marijuana, but he denied other drug use. He had no history of childhood developmental delays, had graduated from high school and was now employed as a security guard. He was an only child, born to nonconsanguineous white parents. His mother was 58 years old with a history of hypertension, and his father was 65 years old and had a history of back pain. There was no family history of stroke, ocular disease, renal disease or muscle cramps.

A computed tomographic (CT) scan of the brain taken immediately on presentation to the emergency department showed an acute deep hemorrhage in the left basal ganglia with surrounding edema (Figure 1A). Computed tomographic angiography was also done at this time to rule out any vascular malformation. No underlying lesion was found. Results of routine blood tests and urine analysis (including a drug screen) were normal. In addition, we ordered a magnetic resonance image of the brain to look for any underlying cause of the hemorrhage (Figure 1B). This image confirmed the presence of an acute deep hemorrhage in the basal ganglia with surrounding edema. There was also diffusely abnormal white matter within the deep white matter of both cerebral hemispheres. Gradient echo imaging, a sensitive sequence for identifying deposition of hemosiderin, showed multiple previous hemorrhages that had been clinically asymptomatic (Figure 1C).

\section{What diagnosis should be considered?}

a. Hypertensive hemorrhage

b. Cerebral autosomal dominant arteriopathy with subcortical infarcts and leukencephalopathy (CADASIL)

c. Cerebral amyloid angiopathy

d. Primary central nervous system vasculitis

e. Drug-induced vasculitis

All of the above potential diagnoses were initially considered (Table 1), but primary central nervous system vasculitis

From the Departments of Clinical Neurosciences and Radiology (Coutts), and Medical Genetics (Innes), University of Calgary, Calgary, Alta.; and the Center for Human Genetics (Matysiak-Scholze, Kohlhase), Freiburg, Germany

CMAJ 2011. DOI:10.1503/cmaj.091496 
or drug-induced vasculitis were our leading considerations. Hypertensive hemorrhage was considered, as this is the commonest cause of hemorrhage in the basal ganglia. However, after a few days of monitoring, this was considered unlikely because there was no suggestion of hypertension. Patients with intracerebral hemorrhage are frequently acutely hypertensive as a response to the hemorrhage, and the absence of this response made underlying hypertension unlikely. CADASIL was a potential diagnosis, given the picture of a young person with substantial white matter disease. However, this was ruled out because patients with CADASIL present with ischemic stroke, not intracerebral hemorrhage. Cerebral amyloid angiopathy was unlikely given that none of the identified hemorrhages were in a lobar location that would be typical in cerebral amyloid angiopathy.

Central nervous system vasculitis is a heterogenous group of conditions characterized by inflammation of blood vessel walls, and can involve both arteries and veins. Accordingly, cerebrospinal fluid was sampled to assess for inflammation, and results of the assessment were normal. The patient underwent full screening of urine and blood for vasculitis (blood tests included complete blood count, creatinine level, antinuclear antibodies, antineutrophil cytoplasmic autoantibodies, erythrocyte sedimentation rate, C-reactive protein, rheumatoid factor, cryoglobulins and complement levels, including $\mathrm{C} 3$ and C4) and the results were negative or within normal limits. Results of a 24-hour urine collection test for protein were also within normal limits.

Our practice in young patients with cerebral hemorrhage is to complete conventional cerebral angiography to rule out rare conditions such as small vascular malformations. In this patient, we suspected cerebral vasculitis as well, and angiog-

Table 1: Main differential diagnoses of intracranial hemorrhage and white matter disease*

\begin{tabular}{|c|c|}
\hline $\begin{array}{l}\text { Type of } \\
\text { condition }\end{array}$ & Diagnosis \\
\hline \multirow[t]{7}{*}{ Vascular } & Hypertension \\
\hline & Cerebral amyloid angiopathy \\
\hline & Cerebral vasculitis \\
\hline & - primary \\
\hline & $\begin{array}{l}\text { - secondary: drug induced, infectious, } \\
\text { systemic vasculitis (e.g., Wegner disease, } \\
\text { polyarteritis nodosa, collagen vascular } \\
\text { diseases), radiation }\end{array}$ \\
\hline & Moyamoya disease \\
\hline & Venous sinus thrombosis \\
\hline \multirow[t]{2}{*}{ Inherited } & CADASIL \\
\hline & COL4A1 mutation \\
\hline Demyelinating & Acute hemorrhagic leukoencephalitis \\
\hline Neoplastic & $\begin{array}{l}\text { Diffuse metastatic brain tumour with } \\
\text { intracerebral hemorrhage }\end{array}$ \\
\hline
\end{tabular}

CADASIL = cerebral autosomal dominant arteriopathy with subcortical infarcts and leukencephalopathy.

*Hypertension and cerebral amyloid angiopathy account for most instances of intracerebral hemorrhage and white matter disease; however, the other rare conditions should be considered depending on the clinical presentation. raphy is the best way of identifying subtle vessel abnormalities. Four-vessel cerebral angiography showed substantial abnormalities in the distal vessels (Figure 2). The distal small arteries of the internal carotid and vertebrobasilar circulations were tortuous and irregular in appearance. The proximal vessels had normal calibre and appearance. There was no beading or dilatation of the proximal vessels that would suggest vasculitis and there was no evidence of an aneurysm or other vascular malformation. The venous structures were normal. This corkscrew appearance of the distal vessels was not typical in appearance for any condition of which we were aware.

After the angiography was completed, it was clear that this patient's condition did not represent any of our original differential diagnoses. The appearance of the distal blood vessels on angiography suggested an abnormality of the small blood vessels in the brain. A skin biopsy was performed to assess the appearance of the small blood vessels. We chose skin as the biopsy site only because of its accessibility. The results of the skin biopsy were normal. Retinal photography was undertaken and this yielded a nonspecific finding of mildly tortuous retinal vessels (Figure 1D). Consultants from rheumatology and nephrology could find no evidence of abnormality outside of the central nervous system.

\section{What is the next step?}

\section{a. Brain biopsy}

b. Presentation at an expert case conference

c. Literature review

This was a challenging case, and, after all the investigations we have described, we still did not have a clear diagnosis. We considered a brain biopsy (a) to directly visualize the tissue, which we hoped would clarify the underlying pathophysiology of this condition (e.g., inflammation, infection), but this would have been very risky in the setting of abnormal blood vessels that had a known tendency to bleed.

We elected to pursue option (b) and presented the case of this patient at our weekly stroke rounds. Although the adult stroke neurologists did not recognize this condition, a pediatric stroke neurologist commented that there is a genetic condition

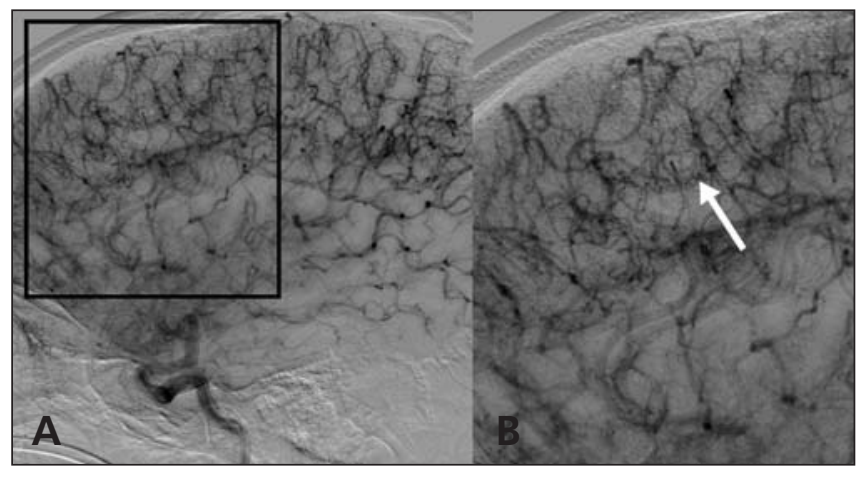

Figure 2: (A) Lateral view, late arterial phase of right internal carotid angiogram. (B) The magnified view shows small, irregular, tortuous distal arterial branches in bilateral carotid and basilar arterial territories (arrow). 
that causes strokes in neonates that he thought could also present in adults. In the absence of a family history of neurologic conditions, we had not considered genetic conditions.

A literature review (c) suggested the possibility of a COLAA1 mutation. The patients with mutations in this gene described in the literature had almost identical imaging to our patient. ${ }^{1,2}$ Although there was no family history of a similar presentation or any neonatal porencephaly (an uncommon condition in which cavities filled with cerebrospinal fluid develop in the brain parenchyma), we considered testing the patient for mutations in $C O L A A 1,{ }^{1,2}$ because these have been reported to cause intracerebral hemorrhage and white matter disease in young adults. One report has described a patient with this gene mutation and sporadic intracerebral hemorrhage. ${ }^{3}$ Our patient was sent for a genetics consultation and arrangements were made for sequencing of the gene COLAAI.

All coding exons and flanking intronic regions of the COLAA1 gene were amplified from the patient's DNA and the polymerase chain reaction products were directly sequenced by routine procedures on an Applied Biosystems 3730XL automated sequencer. The COL4A 1 mutation c.1537-2delA in intron 24 was detected in a heterozygous state in the patient, confirmed on independent investigations by direct sequencing, and subsequently excluded in the patient's parents by direct sequencing. The mutation is predicted to destroy the splice acceptor site of intron 24 . Reverse-transcriptase polymerase chain reaction studies on RNA prepared from peripheral blood lymphocytes showed that the mutation leads to an in-frame deletion of exon 25 in the mRNA (Figure 3).

\section{Discussion}

Stroke is a leading cause of disability, affecting about 1 in 20 people over the age of 14 years in the developed world. Hemorrhagic stroke accounts for $20 \%$ of all strokes. Although some hemorrhagic strokes are caused by vascular malformations, most are caused by abnormalities in the small blood vessels of the brain. There are no good data on what proportion of hemorrhagic stroke is attributable to genetic factors, but a familial pattern is frequently seen.

COLAAl is a gene that encodes the type IV collagen $\alpha-1$ chain. Type IV collagen is present in all basement membranes, including blood vessels. Autosomal dominant mutations in humans have recently been described, although penetrance is incomplete. ${ }^{1,2}$ A wide spectrum of neurologic conditions has been associated with COLAAl mutations, ranging from infantile hemiparesis secondary to perinatal intracerebral hemorrhage (often associated with subsequent porencephaly $\left.{ }^{1}\right)$ to intracranial hemorrhages in adults. ${ }^{2}$ Other associations with COLAA1 mutations include nephropathy, muscle cramps and intracranial aneurysms (our patient had none of these symptoms or conditions). ${ }^{4}$ Imaging findings have included acute intracerebral hemorrhages, microhemorrhages, periventricular leukoencephalopathy, dilated peri vascular spaces, deep porencephaly and brain stem atrophy. ${ }^{1,2}$ Electron microscopy has shown interruptions of the basement membrane of skin capillaries (electron microscopy was not performed on our patient's skin biopsy sample), and tortuosity of the retinal vessels has also been shown. ${ }^{1,2}$ This fits with the hypothesis that $C O L A A 1$ mutations affect small blood vessels, in turn leading to intracerebral hemorrhages.

It is likely that the current literature on COLAAI mutations is biased toward patients with familial patterns and those with striking central nervous system or multisystem involvement (including porencephaly, Axenfeld-Rieger anomaly, retinal artery tortuosity, renal involvement and muscle cramps). As a result, it is likely that these mutations are under-recognized in sporadic cases. Clinical testing of this gene is currently not widely available; however, a frequently updated list of laboratories providing clinical testing for $C O L A A 1$ can be found at the GeneTests website. ${ }^{5}$ Access to clinical genetic testing varies among Canadian provinces, ${ }^{6}$ and most jurisdictions

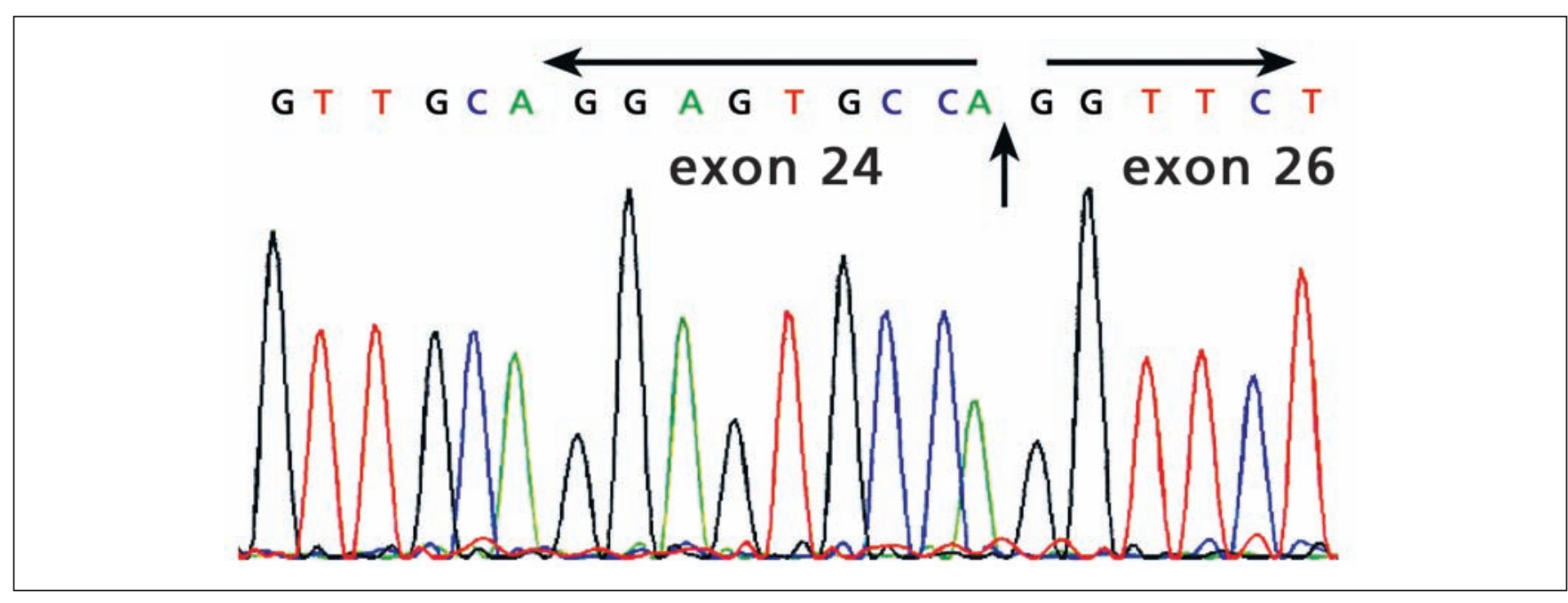

Figure 3: DNA sequencing of a COL4A1 complementary DNA product obtained by reverse-transcriptase polymerase chain reaction shows lack of exon 25 sequence between exon 24 and exon 26 resulting from the mutation c.1537-2delA in intron 24 of the COL4A1 gene. 
have provisions to secure such testing if it affects the clinical management or genetic counselling of the patient and family.

COL4A1 mutations should be considered in any young patient with an intracerebral hemorrhage. The presence of a positive family history, or other features including white matter disease, ocular disease, renal disease or muscle cramps should prompt a decision for genetic testing.

The mutation in this patient was a splice site mutation resulting in an in-frame deletion of exon 25 of the COLAAI mutation. The in-frame deletion in our patient leads to a deletion of 64 amino acids within the triple helical region of COL4A1. Other reported missense mutations in this gene affect glycine residues within the same region, and it is anticipated that our patient's mutation would similarly affect stability of collagen bundles. It is possible that mutations in other regions of the gene may result in variable phenotypes in addition to those reported so far, analogous to other collagens such as $C O L 2 A 1$, where different mutations have resulted in a wide range of phenotypes, including prenatal lethal skeletal dysplasias and isolated familial retinal disease.

\section{Patient outcome}

Subsequent to our patient's presentation, his mother experienced a subarachnoid hemorrhage, and was ultimately identified as having intracerebral aneurysms. As aneurysms have been reported in COL4AI families, she may have been a mutation carrier. However, results of direct sequencing for the mutation in both parents were normal, confirming a de novo mutation in COLAA1. There are several explanations for the occurrence of an autosomal dominant condition in a person with a negative family history. The most likely is often a de novo mutation. For example, in the autosomal dominant condition type 1 neurofibromatosis, nearly half of all affected people have a de novo mutation. Given that sequencing of COLAA1 so far has been limited primarily to familial cases, it is likely that other de novo mutations in this gene will be found in sporadic cases. Other explanations for a negative family history could include mosaicism in a parent, nonpaternity and reduced penetrance.

Our patient has done well since discharge from hospital, with no further clinical events. However, repeat magnetic resonance imaging six months after our patient's initial presentation showed new areas of deep microhemorrhage that had been clinically asymptomatic. A recent report describes the long-term follow-up of a family with a COLAA1 mutation. None of this particular family had any recurrent events or new gradient echo lesions on magnetic resonance imaging after the index event, but within the family there was great diversity of clinical expression.?

In our patient, we have seen evidence of ongoing asymptomatic hemorrhaging within the brain. Only long-term followup will give us an idea of his prognosis. Previous reports have described the role of minor trauma in intracerebral hemorrhage in patients with the COLAA1 mutation. Based on this limited evidence, we have advised our patient to take special care to avoid any minor injuries to the head, including avoiding contact sports that may contribute to further hemorrhages.

This article has been peer reviewed.

Competing interests: None declared.

Acknowledgements: The authors acknowledge Drs. Adam Kirton and Nic Weir for their suggestions, Dr. Mark Hudon for help with imaging descriptions and Chandree Beaulieu for technical assistance.

\section{REFERENCES}

1. Gould DB, Phalan FC, van Mil SE, et al. Role of COL4A1 in small-vessel disease and hemorrhagic stroke. N Engl J Med 2006;354:1489-96.

2. van der Knaap MS, Smit LM, Barkhof F, et al. Neonatal porencephaly and adult stroke related to mutations in collagen IV A1. Ann Neurol 2006;59:504-11.

3. Vahedi K, Kubis N, Boukobza M, et al. COL4A1 mutation in a patient with sporadic, recurrent intracerbral hemorrhage. Stroke 2007;38:1461-4.

4. Plaisier E, Gribouval O, Alamowitch S, et al. COL4A1 mutations and hereditary angiopathy, nephropathy, aneurysms and muscle cramps. N Engl J Med 2007; 357:2687-95.

5. GeneTests [homepage]. Bethesda (MD): National Center for Biotechnology Information; 2010. Available: www.ncbi.nlm.nih.gov/sites/GeneTests/?db=GeneTests (accessed 2009 Nov. 13)

6. Silversides A. The wide gap between genetic research and clinical needs. CMAJ 2007; 176:315-6.

7. Vahedi K, Boukobza M, Massin P, et al. Clinical and brain MRI follow-up study of a family with COL4A1 mutation. Neurology 2007;69:1564-8.

Correspondence to: Dr. Shelagh Coutts, Department of Clinical Neurosciences, Rm. C1246, Foothills Medical Centre, 1403 29th St. NW, Calgary AB T2N 2T9; shelagh.coutts@calgaryhealthregion.ca

\section{Resources}

\section{Articles}

- Rost NS, Greenberg SM, Rosand J. The genetic architecture of intracerebral hemorrhage. Stroke 2008;39:2166-73.

- Greenberg SM. Small vessels, big problems. N Engl J Med 2006;354:1451-3.

\section{Websites}

- Online Mendelian Inheritance in Man: www.ncbi.nlm.nih.gov/omim/

Online Mendelian Inheritance in Man is a comprehensive, authoritative and timely compendium of human genes and genetic phenotypes. This database is searchable and updated frequently. A main focus of this site is the relationship of genotypes to phenotypes.

- GeneTests and GeneReviews: www.ncbi.nlm.nih.gov/sites/GeneTests/?db = GeneTests The GeneTests website is a publicly funded medical genetics information resource developed for physicians, other health care providers and researchers, available at no cost to all interested persons. The laboratory directory at GeneTests provides an international searchable resource of clinical and research laboratories providing genetic testing for different disorders. The companion GeneReviews provides expert-authored, peer-reviewed descriptions of genetic diseases. A GeneReview on conditions related to COL4A1 is available at www.ncbi.nlm .nih.gov/bookshelf/br.fcgi?book = gene\&part = col4a1-dis. 